\title{
Robust and conductive hydrogel based on mussel adhesive chemistry for remote monitoring of body signals
}

\author{
Weijun LI ${ }^{1, \dagger}$, Hao LIU ${ }^{1, \dagger}$, Yuanyuan MI ${ }^{1}$, Miaoran ZHANG $^{1}$, Jinmiao SHI ${ }^{1}$, Ming ZHAO $^{1}$, Melvin A. \\ RAMOS $^{2}$, Travis Shihao $\mathbf{H U}^{2}$, Jianxiong $\mathbf{L I}^{3}$, Meng $\mathbf{X U}^{3}$, Quan $\mathrm{XU}^{1, *}$ \\ ${ }^{I}$ State Key Laboratory of Heavy Oil Processing, Beijing Key Laboratory of Biogas Upgrading Utilization, Harvard SEAS- \\ CUPB Joint Laboratory on Petroleum Science, China University of Petroleum, Beijing 102249, China \\ ${ }^{2}$ Department of Mechanical Engineering, California State University, Los Angeles, CA 90032, USA \\ ${ }^{3}$ Department of Orthopedics General Hospital of Chinese People's Liberation Army, Beijing 100853, China \\ Received: 20 May 2020 / Revised: 01 June 2020 / Accepted: 09 June 2020 \\ (C) The author(s) 2020 .
}

\begin{abstract}
There is a high demand for hydrogels with multifunctional performance (a combination of adhesive, mechanical, and electrical properties) in biological, tissue engineering, robotics, and smart device applications. However, a majority of existing hydrogels are relatively rigid and brittle, with limited stretchability; this hinders their application in the emerging field of flexible devices. In this study, cheap and abundant potato residues were used with polyacrylamide (PAM) to fabricate a multifunctional hydrogel, and chitosan was used for the design of a three-dimentional (3D) network-structured hydrogel. The as-prepared hydrogels exhibited excellent stretchability, with an extension exceeding $900 \%$ and a recovery degree of over $99 \%$. Due to the combination of physical and chemical cross-linking properties and the introduction of dopamine, the designed hydrogel exhibits a remarkable self-healing ability $(80 \%$ mechanical recovery in $2 \mathrm{~h}$ ), high tensile strength $(0.75 \mathrm{MPa})$, and ultra-stretchability $(900 \%)$. The resultant products offer superior properties compared to those of previously reported tough and selfhealing hydrogels for wound adhesion. Chitosan and potato residues were used as scaffold materials for the hydrogels with excellent mechanical properties. In addition, in vitro experiments show that these hydrogels feature excellent antibacterial properties, effectively hindering the reproduction of bacteria. Moreover, the ternary hydrogel can act as a strain sensor with high sensitivity and a gauge factor of 1.6. The proposed strategy is expected to serve as a reference for the development of green and recyclable conductive polymers to fabricate hydrogels. The proposed hydrogel can also act as a suitable strain sensor for bio-friendly devices such as smart wearable electronic devices and/or for health monitoring.
\end{abstract}

Keywords: bionic mussel; hydrogel; waste potato residues; adhesion; conductivity

\section{Introduction}

Polymer hydrogels can integrate multiple functions in one design, including toughness, viscosity, selfhealing, electrical conductivity, and responsiveness, thus widening the range of applications in tissue engineering, electronic skin, wound healing, drug release, and soft robotics [1,2]. An increasing number of skin-attached wearable materials is being invented to match the rapid development of wearable devices. These materials can easily adhere to the skin for health monitoring in real time. However,

$\uparrow$ These authors contributed equally to this work.

* Corresponding author: Quan XU, E-mail: xuquan@cup.edu.cn 
the majority of mainstream wearable devices are still limited to commonly used watches and glasses that do not often have the flexibility and stretchability to adhere directly to the skin. Therefore, designing more flexible, stretchable, and stable adhesive materials can widen the range of applications [3-6]. In addition to strong adhesiveness, the most essential properties for excellent hydrogels include flexibility, recoverability, and environmental stability. Recently, multifunctional hydrogels including interpenetrating network hydrogels [7, 8], double network hydrogels [9-12], and nanocomposite hydrogels [13-15], have been developed. For example, Han et al. [16] prepared hydrogels by introducing polydopamine (PDA) nanoparticles into polyacrylamide (PAM) and clay networks to form PDA-clay-PAM hydrogels. Since the catechol group on PDA is analogous to dihydroxyphenylalanine (DOPA), which is the main residue of mussel silk adhesion protein, it plays a vital role in achieving mussel-like adhesion. Adding PDA can provide materials with good tissue adhesion and cell affinity. However, a prominent defect of general dopamine-containing hydrogels is that the two hydroxyl groups on the catechol group are easily oxidized, and the adhesive properties of the material are gradually lost. In addition, the reaction conditions are very harsh during the preparation of dopamine-containing hydrogels [17]. For example, when the percentage of dopamine is high, strong cross-linking between the dopamine and polymer cannot be formed making the gelation process difficult [17-20]. Therefore, the dopamine content and the degree of cross-linking significantly limit the adhesion strength of hydrogels. Hence, many researchers have made significant efforts to develop strong bonding materials to address this problem. For example, Liu et al. developed an adhesive hydrogel with enhanced nucleobases that can be bonded through hydrogen bonding, hydrophobic interactions, metal complexes, $\pi-\pi$ stacking, and cation- $\pi$ interactions. Such interactions allow the materials to adhere to various solid surfaces and biological tissues [21]. A recent integration of polydopamine-coated talc nanosheets into a PAM hydrogel modeling mussel adhesive chemistry has been implemented successfully. This hydrogel shows improved mechanical properties, adhesiveness, biocompatibility, and conductivity [22]. However, the mechanical properties are still below expectations compared to the industrial requirements. Therefore, there is an urgent need to develop a cheap high-performance hydrogel material.

The present work demonstrates that waste potato residue powders can be used as an additive to improve the performance of the hydrogels because they contain a large amount of super-hydrophilic cellulose, starch, and pectin. Furthermore, potato residues also carry a lot of positive charges that can provide the composite hydrogel materials with a stable frame and enhanced performance. Because of the hydrophilic frame structure, waste potato residues (S) are suitable for fabricating hydrogel materials with the desired properties [23, 24]. Therefore, we used cheap and readily available potato pomace as the raw material for the preparation of hydrogels while also reducing environmental pollution. Specifically, we have developed a ternary network hydrogel that integrates chitosan (C), S, and PDA into chemically cross-linked PAM to obtain polydopamine-chitosan-waste potato residuespolyacrylamide (PDA-C-S-PAM) hydrogel. The threenetwork structure of chitosan and acrylamide was introduced simultaneously to prepare a multifunctional hydrogel material. $\mathrm{C}$ and $\mathrm{S}$ acted as scaffolds that provided the hydrogel with excellent mechanical properties. In addition, due to the introduction of PDA, the hydrogel also exhibited excellent adhesive and self-healing properties [9, 25-27]. The mechanical strength of the PDA-C-S-PAM hydrogel was as high as $0.75 \mathrm{MPa}$. Furthermore, the original resistance and the tensile properties could be restored after $120 \mathrm{~min}$ of repair that is critical for widening its industrial applications and reducing the negative environmental impact. In addition, this hydrogel was also found to exhibit an excellent electrical conductivity. In vitro experiments further showed that the antibacterial rate of the hydrogel PDA-CS-PAM against Escherichia coli (E.coli) reached $81.6 \%$, thereby showing good antibacterial properties. The fabricated hydrogel was also found to integrate several desirable characteristics including superadhesiveness, high toughness, self-repairing capability, 
conductivity, and antibacterial properties that increase its applicability in designing electronic wearable devices for monitoring human physiological and other health conditions.

\section{Experimental section}

\subsection{Materials}

Dopamine hydrochloride (DA) was acquired from Shanghai Anaiji Chemical Co., Ltd. Chitosan was obtained from Bellingway Technology Co., Ltd. Ammonium persulfate (APS) and acrylamide (AM) were obtained from Tianjin Fuchen Chemical Reagent Co. N,N'-methylenebisacrylamide (BIS) was obtained from Shanghai Aladdin Reagent Co., Ltd. Sodium hydroxide and acetic acid used in the experiment were purchased from Beijing Reagent Co., Ltd. Waste potato residues, potato skins, or waste potatoes were recovered from local restaurants. The collected potato residues were dried at $80{ }^{\circ} \mathrm{C}$, ground into powder, and sieved using a 60-mesh stainless steel mesh to obtain the required potato flour residues. All chemicals and reagents were used directly in the experiments without further processing. LB (Lysogeny broth) medium, DMEM (Dulbecco's modified eagle medium) (ATCC, USA); fetal bovine serum (FBS) (ICN Biomedicine, Germany); Cell-Counting Kit-8 reagent (CCK8) (Dojindo, Japan); $\mathrm{CO}_{2}$ constant temperature incubator (SANYO, Japan); optical microscope (OLYMPUS, Japan); microplate reader RT-6000 (Bio-Rad, USA); cell culture plate (Costar, USA).

\subsection{Preparation of hydrogel}

(1) First, 1\% waste potato residue solution and 2\% chitosan solution were added to $10 \mathrm{~mL}$ of deionized water while stirring; then, different amounts of dopamine hydrochloride were added. The solution was magnetically stirred at $25{ }^{\circ} \mathrm{C}$ for $10 \mathrm{~min}$ to mix them completely. Sodium hydroxide solution $(1 \mathrm{M})$ was then added to adjust the solution $\mathrm{pH}$ to 9, and stirring continued for $30 \mathrm{~min}$ so that DA was self-polymerized while stirring in air. (2) Acrylamide (AM), ammonium persulfate (APS), and N,N'-methylenebisacrylamide (BIS) were mixed with the PDA solution while stirring. After $5 \mathrm{~min}$ of mixing, the stirrer was removed to allow AM polymerization to form a composite hydrogel. The obtained hydrogel was washed three times with deionized water to remove surface residues. Hydrogels with different mass ratios (including DA/AM and $\mathrm{C}-\mathrm{S} / \mathrm{AM}$ ) were synthesized using the same experimental steps to study the effect of different amounts of substances on the properties of the hydrogel. The different proportions of added substances are provided in Table S1 in the Electronic Supplementary Material (ESM). In the experiment, more initiator APS was consumed when DA was present than when DA was absent. In addition, it was found that the ratio of DA/AM was not higher than $0.8 \mathrm{wt} \%$. Otherwise, the composite gel could not be successfully obtained. Consistent with the results reported in the previous studies, the presence of reducing DA molecules was found to affect the APS activity, thereby reducing the polymerization of AM monomers.

\subsection{Fourier transform-infrared spectrometer (FT- IR) analysis}

The test samples were dried using a freeze dryer, and the FT-IR spectra of pure PAM, C-S-PAM, PDA-PAM, and PDA-C-S-PAM hydrogels were tested using an FT-IR spectrometer (Nicolet IS 10, Thermo, USA), in which the spectral range was between 4,000 and $500 \mathrm{~cm}^{-1}$. The test was performed in the attenuate total reflectance (ATR) mode.

\subsection{Ultraviolet ray (UV) analysis}

Ultraviolet absorption curve was measured using a UV spectrophotometer (UV-9000, Metash, China). The UV absorption intensity of DA and PDA-C-S solutions with optimal concentration was tested. The test solution needed to be diluted 5 times with deionized water.

\subsection{X-ray photoelectron spectroscopy (XPS) analysis}

XPS (Thermo escalab 250Xi, Thermo, USA) was used to measure the surface chemical composition of the hydrogels. In the test, the excitation source was monochromatic $\mathrm{K} \alpha$ X-rays $(\mathrm{h} v=1,486.6 \mathrm{eV})$. The neutral C1s peak (set to $285.0 \mathrm{eV}$ ) needed to be 
corrected as the reference charge. Before each test, the sample was washed three times with deionized water to remove surface residues, and then the sample was dried using a freeze dryer for $12 \mathrm{~h}$ to obtain a test sample.

\subsection{Scanning electron microscopy (SEM) charac- terization}

Scanning electron microscopy (SEM, Hitachi SU 8220, Hitachi, Japan) (DA/AM=0.8 wt $\% \mathrm{CS} / \mathrm{AM}=$ $0.4 \mathrm{wt} \%$ ) was used to characterize the morphology of the PAM, C-S-PAM, PDA-PAM, and PDA-C-SPAM hydrogels. Before scanning, the hydrogel needed to be freeze-dried using a freeze dryer. Then, a portion of the hydrogel was cut to expose its internal structure for easy observation.

\subsection{Mechanical test}

Tensile tests of the specimens were performed using a universal testing machine (Instron 5567, Norwood, USA). The loading speed was set to $50 \mathrm{~mm} \cdot \mathrm{min}^{-1}$ at room temperature. The specimen was $30 \mathrm{~mm}$ long, $20 \mathrm{~mm}$ wide, and $5 \mathrm{~mm}$ thick, and the cross section was rectangular. Each sample was tested three times, and the average value was taken as the final data. The tensile strain was normalized from the original length (10), and the tensile stress was normalized from the cross-section of the original sample. The general elongation was calculated by determining the ratio of the length after deformation (1) to the original length (10).

\subsection{Adhesive strength test}

The adhesion strength of hydrogels on the surfaces of various materials was measured using a universal testing machine in the $0^{\circ}$ peeling mode. Standard samples were prepared with a length of $30 \mathrm{~mm}$, width of $20 \mathrm{~mm}$, and thickness of $5 \mathrm{~mm}$. Fresh pigskin (purchased from a local mall) was bonded to a glass sheet with a cyanoacrylate adhesive as a hard backing for the hydrogel adhesives. The prepared hydrogel samples were then attached between two pieces of pigskin. These samples were then added between two pieces with the attached pigskin. The detachment process was tested at a constant $0^{\circ}$ peel speed of $50 \mathrm{~mm} / \mathrm{min}$. Various substrate materials were also tested, including agate, glass, ceramic, alumina, polytetrafluoroethylene (PTFE), polyethylene, rubber, wood blocks, aluminum, nickel alloys, copper-tin alloys, brass, magnets, stainless steel, and animal tissues. The tests were repeated at least five times for each sample to determine the peeling strength.

\subsection{Conductivity test}

The hydrogel samples (size $30 \mathrm{~mm} \times 20 \mathrm{~mm} \times 5 \mathrm{~mm}$ ) were tested with an electrochemical workstation (Solartron SI 1287/1260, Solartron ISA, UK) to determine its electrical conductivity and other electrical properties using different tensile materials. To measure the change in resistance, the specific tensile strains/strain rates were implemented using the following protocol where the specific tensile strains/ strain rates could be controlled ranging from $1 \%$ to $900 \%$, with the times controlled between 10 and $60 \mathrm{~s}$. Each sample was measured three times, and the average value was taken as the final representative result. In each test, a constant standard voltage $(V)$ of $5 \mathrm{~V}$ was used, and the change in the current $(I)$ was recorded. The electrical resistance $(R)$ was calculated according to Ohm's law $(R=U / I)$.

\subsection{In vitro antimicrobial assay}

The PDA-C-S-PAM hydrogel (8 $\mathrm{mm}$ in diameter and $0.5 \mathrm{~mm}$ in thickness) was washed three times using sterile deionized water and PBS to remove unreacted monomers, crosslinkers, and initiators in the hydrogel. The prepared samples were subjected to aseptic treatment with $60 \mathrm{Co}-\gamma$ ray sterilization. The pretreated sample was placed in a 96-well cell culture plate, $100 \mu \mathrm{L}$ of bacterial suspension (106 CFU/mL) was added dropwise to the surface of the hydrogel, and incubated at $37{ }^{\circ} \mathrm{C}$ for $12 \mathrm{~h}$. After incubating for $12 \mathrm{~h}, 900 \mu \mathrm{L}$ of LB liquid medium was added to each sample, and the incubation continued at $37{ }^{\circ} \mathrm{C}$ for $12 \mathrm{~h}$. Finally, $200 \mu \mathrm{L}$ was taken from the bacterial suspension in each sample, transferred to a 96-well cell culture plate, and the absorbance (OD) of the bacterial suspension was analyzed using a microplate reader, with the wavelength set at $600 \mathrm{~nm}$. 


\section{Results and discussion}

\subsection{Multifunctional hydrogel design process}

One percent waste potato residue solution, two percent chitosan solution, and PDA were added to the acrylamide to prepare a highly adhesive, selfrepairing, and biocompatible hydrogel. As shown in Fig. 1, dopamine is first pre-loaded on the chitosan and waste potato residue chains under alkaline conditions and polymerized to form a prepolymer PDA-C-S. The acrylamide (AM) monomer, initiator ammonium persulfate (APS), cross-linking agent BIS, and accelerator tetramethylethylenediamine (TEMED) were added to the above pre-polymer to form a composite hydrogel PDA-S-C-PAM (as shown in Figs. 1(a) and 1(b)). Physical and chemical crosslinking in the hydrogel was observed (as shown Fig. 1(c)). Physical cross-linking constitutes of weak forces such as hydrogen bonding, $\pi-\pi$ conjugation, and chain entanglement, whereas covalent crosslinking enhances the stability of the hydrogel. Cross-linking increases the mechanical and thermal stability of the hydrogel. PAM is cross-linked with waste potato residues and chitosan through a pretreatment process involving a non-covalent reaction, providing the basic framework for the hydrogel. The main PAM chain provides strength to the network structure during the stretching process. In addition, PDA contains numerous catechols, an analog of mussel adhesion protein to obtain a hydrogel with superior cell affinity and tissue adhesion to the aqueous bulk. Furthermore, because of the presence of free electrons (as a result of $\mathrm{Na}^{+}$, $\mathrm{K}^{+}$present in the potatoes), the prepared hydrogel has good conductivity without the need for additional conductive components. This strategy also offers a convenient approach to obtain similar properties as those of other conductive functional materials.

\subsection{Characterization of multifunctional hydrogels}

To establish the source of this versatile hydrogel performance, we performed a series of analyses to characterize the hydrogel materials including scanning electron microscopy (SEM), Fourier transform-infrared (FT-IR), ultraviolet-visible (UV-vis) spectrometry, and X-ray photoelectron spectroscopy (XPS). It was concluded that PDA-C-S is responsible for the self-healing and adhesion properties of the hydrogel and also contributes to the high stretchability. The microstructure of hydrogel was analyzed by means of SEM and the results are shown in Figs. 2(a)-2(d) that indicate the microscopic topography of PAM, CS-PAM, PDA-PAM, and PDA-C-S-PAM, respectively. It is evident that there are many hollow structures in the microscopic morphology of the hydrogel. The

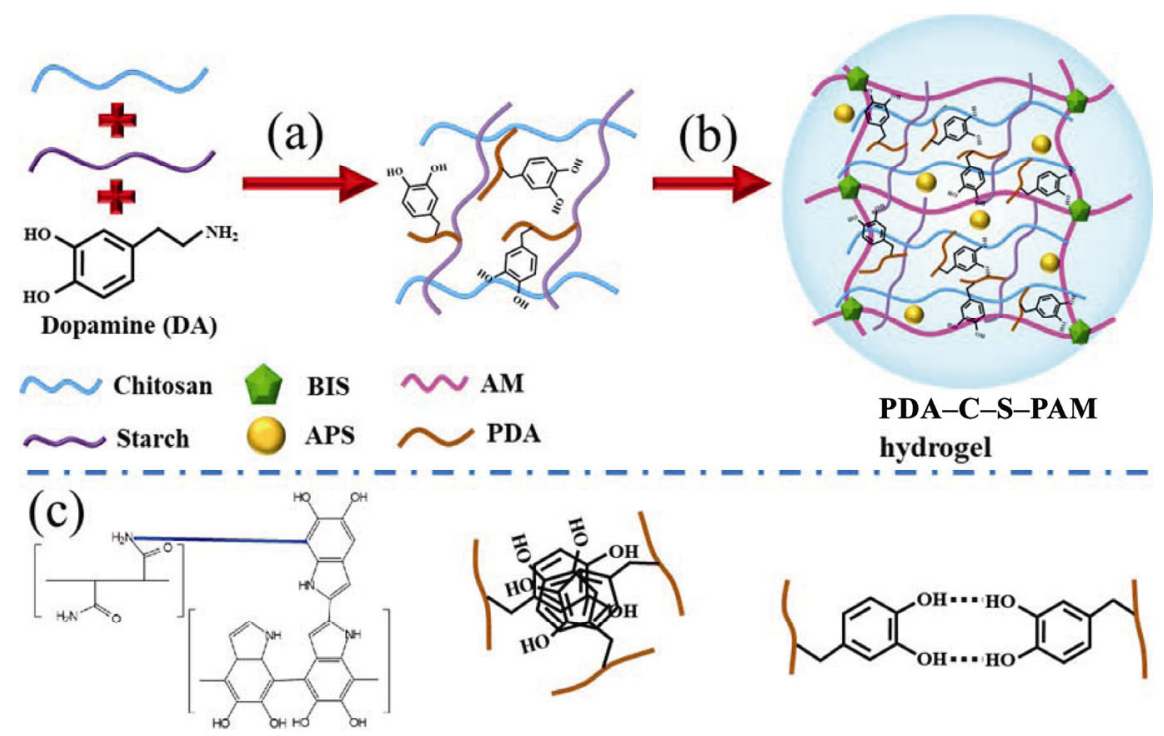

(1) Interactions

(2) $\pi-\pi$ stacking

(3) Hydrogen bonds

Fig. 1 (a, b) Mechanism of preparation of PDA-C-S-PAM. (c) Internal interactions of the materials. 
hollow structure of these bodies contains several fine pore structures (as shown in Fig. 2(d)), and no significant porous structures are found in the other three types of hydrogels (as shown in Figs. 2(a)2(c)). This fine pore structure is responsible for the material's super-strong mechanical properties. According to Ref. [16], pure PAM has a smooth surface structure, whereas composite hydrogels exhibit a certain porous and microfibrous structure. The formation of microfibers may be due to the interaction between PDA-C-S and PAM since dopamine (DA) can form intermolecular interactions with many polymers, thereby affecting the polymerization of monomers and materials during the polymerization process. In addition, there were no significant multiple microstructures in pure PAM, C-S-PAM, and PDAPAM. For PDA-C-S-PAM, multiple microstructures (as shown in Fig. 2(d)) were clearly observed. This microstructure extends from the basic microscopic framework and provides further connectivity and support for the framework that enhances the mechanical properties of the material as well as other properties. The basic color of PAM material is also observed to change significantly due to the addition of PDA. Figure S1 in the ESM shows digital photos of the aforementioned four hydrogel materials, and the transparent color of the composite material is observed to turn brown on addition of DA.

FT-IR spectroscopy was also used to further characterize the material in order to systematically study the bonding mode inside the material. The
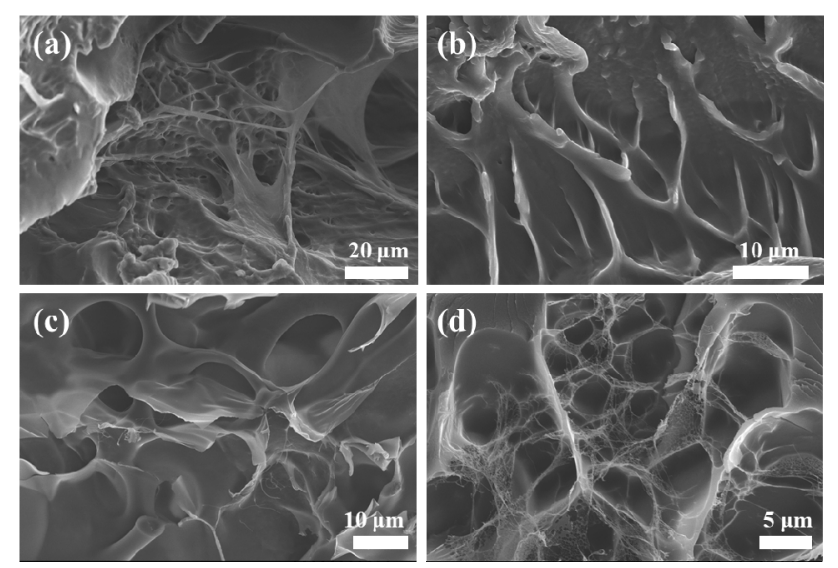

Fig. 2 SEM images of (a) PAM, (b) C-S-PAM, (c) PDAPAM, and (d) PDA-C-S-PAM.

results are shown in Fig. 3(a). The infrared absorption image indicates that the composite hydrogel and other types of hydrogels exhibit remarkably similar curve patterns, as compared to several other hydrogels. The peak displays a characteristic trend where the hydroxyl peaks in the material are significantly enhanced by the addition of starch and chitosan. In addition, the presence of some methyl peaks shows an increase in the addition of starch and chitosan. The tensile vibration of the $\mathrm{C}=\mathrm{O}$ group of the amide and $\mathrm{CH}_{2}$ corresponds to the interval between 1,648 and 1,448 $\mathrm{cm}^{-1}$, and the band between 2,922 and $3,500 \mathrm{~cm}^{-1}$ corresponds to the $\mathrm{NH}$ bond of the stretching vibration, indicating successful synthesis (as shown in Fig. S2 in the ESM). For PAM, a small peak observed at $1,320 \mathrm{~cm}^{-1}$ indicates a vibration corresponding to $\mathrm{C}-\mathrm{N}$ bond stretching of aniline on the hydrogel, suggesting an interaction between PAM and the $\mathrm{NH}_{2}$ group in catechol. Dopamine and potato residues were also introduced, and an enhancement in the peak of the benzene ring was observed (as shown in Fig. S2(c) in the ESM). We also used UV absorption spectra to further characterize the material. As a result, a distinct peak was detected at $308 \mathrm{~nm}^{-1}$ (as shown in Fig. 3(b)), indicating the formation of dehydrodopamine at this point.

The XPS high-resolution C1s curve of PDA-C$\mathrm{S}-\mathrm{PAM}$ is shown in Fig. 3(c) that shows a $\mathrm{C}-\mathrm{O}$ bond at $285.8 \mathrm{eV}$ and a $\mathrm{C}=\mathrm{O}$ bond at $288.1 \mathrm{eV}$ on the curve belonging to the catechol and anthracene groups in the material, respectively. The statistical results of XPS data (Fig. 3(d)) show that there is still a higher proportion of $\mathrm{C}-\mathrm{O}$ groups in PDAC-S-PAM, indicating that a large amount of catechol groups is retained in the material. In contrast, no $\mathrm{C}=\mathrm{O}$ group was detected on dopamine, as shown in Fig. S3(a) in the ESM and Fig. 3(d). Similarly, the high-magnification XPS and XPS full-spectrum curves for O1s and N1s are given in Fig. S3 in the ESM.

\subsection{Mechanical properties of the hydrogels}

Figure 4(a) shows the optical image of a PDA-CS-PAM hydrogel sample with an initial length of $25 \mathrm{~mm}$ stretched to the length of $110 \mathrm{~mm}$. According to the typical stress-strain curve (as shown in Fig. $4(\mathrm{c})$ ), the PDA-C-S chain plays a crucial role in 
(a)
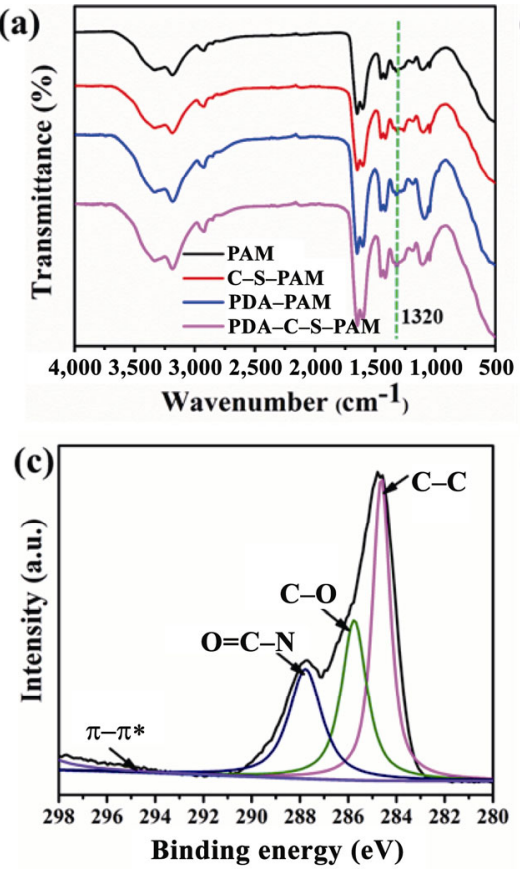

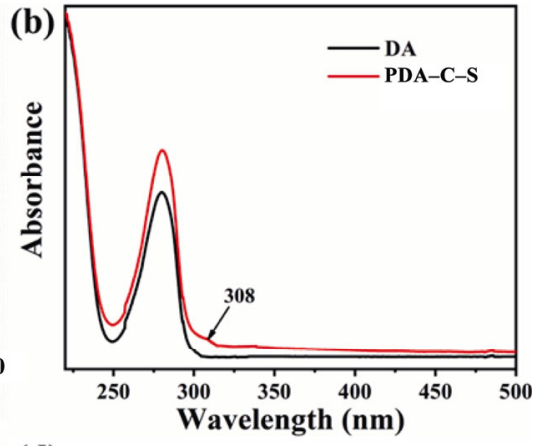

(d)

\begin{tabular}{cccc}
\hline $\begin{array}{c}\text { Peak } \\
\text { species }\end{array}$ & $\begin{array}{c}\text { Binding } \\
\text { energy }\end{array}$ & DA & $\begin{array}{c}\text { PDA-C- } \\
\text { S-PAM }\end{array}$ \\
\hline $\mathbf{C}-\mathbf{C}$ & 284.6 & $48.6 \%$ & $60.4 \%$ \\
$\mathbf{C}-\mathbf{O}$ & 285.7 & $15.9 \%$ & $22.5 \%$ \\
$\mathbf{C}-\mathbf{N}$ & 288.1 & $31.2 \%$ & $14.4 \%$ \\
$\mathbf{C}=\mathbf{O}$ & 288.1 & - & $2.1 \%$ \\
$\pi-\pi *$ & 291.3 & $4.3 \%$ & $0.6 \%$ \\
\hline
\end{tabular}

Fig. 3 (a) FT-IR spectra of PAM, C-S-PAM, PDA-PAM, and PDA-C-S-PAM. (b) UV-vis spectra of DA and PDA-C-S. (c, d) XPS spectra of the hydrogel.

(a)

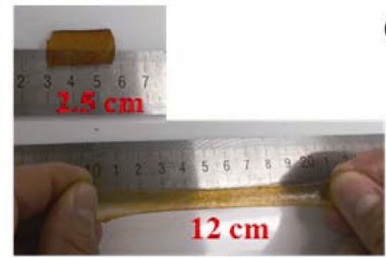

(c)

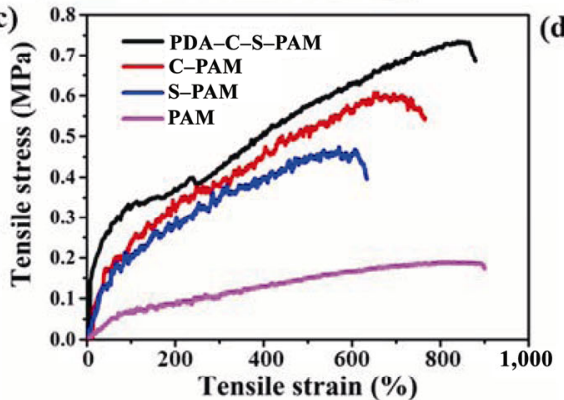

(e)

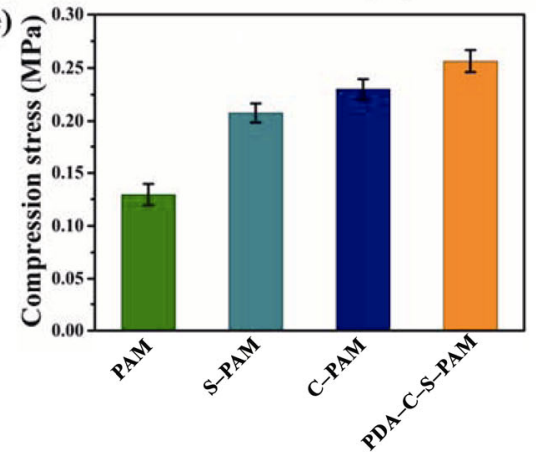

(b)

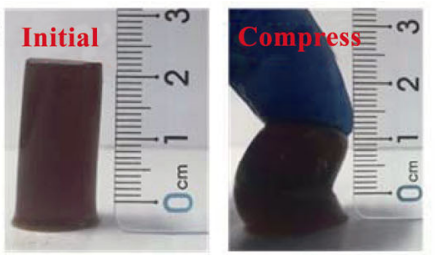

(d)
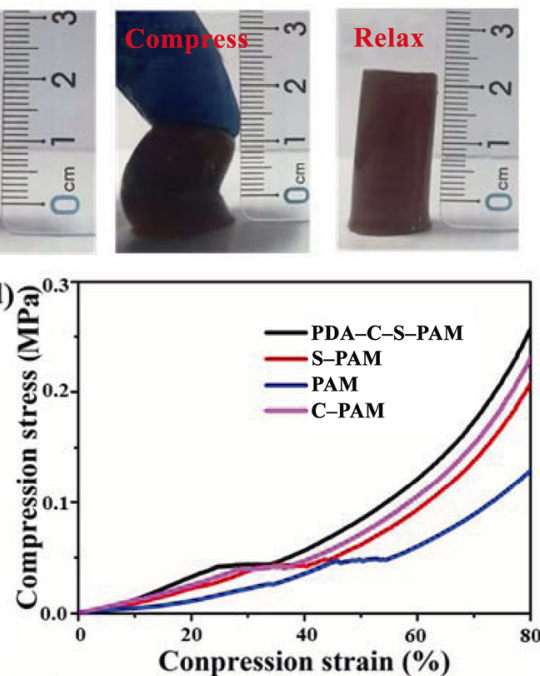

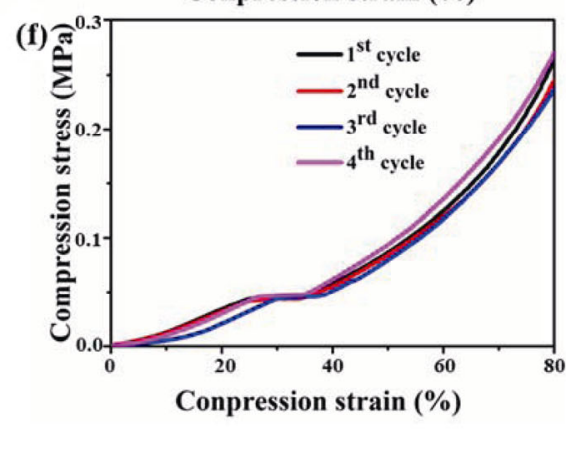

Fig. 4 (a) Tensile performance of PDA-C-S-PAM hydrogel under external force. (b) High recovery performance of hydrogels under external force. (c) Tensile strength. (d) Compression strength. (e) Detailed pressure data of hydrogels. (f) Pressure data after four cycles. 
achieving high tensile strength and super-stretchability. The compressive properties of the material were also tested. As shown in Fig. 4(b), the original material can reach a height of $26 \mathrm{~mm}$. In presence of an external force, the height of the sample is compressed as much as possible. After withdrawing the compression pressure, it returns to its original height within a few seconds indicating that the material has excellent compression resistance. The overall mechanical tensile properties of the material are shown in Fig. 4(c). It is concluded that the composite hydrogel material has improved tensile properties with the tensile strength reaching a maximum of $0.75 \mathrm{MPa}$ at a tensile strain of $900 \%$. This ultra-high tensile property of the composite outperforms that of the other comparative samples (the maximum tensile strength of C-PAM is $0.609 \mathrm{MPa}$ at a maximum tensile strain of $730 \%$ ). The tensile strength of the PDA-PAM hydrogel reached up to $0.81 \mathrm{MPa}$ at a tensile strain of $560 \%$ (as shown in Fig. S4(a) in the ESM). The tensile strain of the C-SPAM hydrogel also reached $900 \%$, but a lower tensile strength of $0.63 \mathrm{MPa}$ (as shown in Fig. S4(a) in the ESM). In short, both the addition of the potato residue and the interpenetration of chitosan contribute to the excellent mechanical properties of the hydrogel. First, after the potato residue was added, the strain at break was lowered remarkably, and the mechanical strength was improved significantly. Second, the mechanical strength of the C-PAM hydrogel was found to increase with increasing chitosan content. The hydrogel exhibits good mechanical properties due to the synergistic effect of chitosan interpenetration and chitosan enhancement. Further compression tests have shown that the hydrogel can recover quickly under $80 \%$ compression (Fig. 4(d)), and the maximum pressure it can withstand reaches $0.252 \mathrm{MPa}$, which is significant compared to pure PAM (0.159 MPa). Furthermore, the addition of chitosan and potato residues resulted in a significant improvement in the compressive properties of PAM, but this was still lower compared to that of composite hydrogel materials. Cyclic tests indicated that the hydrogel could recover automatically under greater deformation (80\%).

\subsection{Excellent adhesion of the hydrogels}

The composite hydrogel material has excellent adhesion and can firmly adhere to various surface types. As shown in Fig. S5 in the ESM, the hydrogel material can easily adhere to ceramics, glasses, carnelian alumina, PTFE, polythene, rubbers, plastics, wood, aluminum, nickel alloy, copper-zinc alloy, brass, magnetite, stainless steel, and tissues. The above composite hydrogel materials are expected to be applied to a variety of fields such as tissue adhesives, adhesive electronic devices, and portable instruments. The material adhesion test is schematically illustrated in Fig. 5(a). The hydrogel is sandwiched between two sheets of glass with pigskin to form a sandwich structure that is stretched by an external force until separation. This structure was found to exhibit adhesion properties similar to that of the hydrogel material. Notably, we found that after 50 cycles of adhesion testing, the adhesion of the hydrogel remained above $18 \mathrm{kPa}$ (as shown in Fig. 5(b)). This excellent adhesion also resulted in different surfaces that were previously tested. The test was carried out, and the results are shown in Fig. 5(c). As the concentration of dopamine, chitosan, and potato residue increased, the adhesion property of the hydrogel also increased. To verify this observation, we selected two samples. DA/AM $=0.8 \%$ and CS/ $\mathrm{AM}=0.4 \%$ were used to carry out further experiments. At the same time, we also tested the durability of the PDA-C-S-PAM hydrogel and the effect of different surfaces on adhesion. As shown in Fig. 5(d), the adhesion strength between the PDAC-S-PAM hydrogel and various materials gradually increased over time, and the adhesion performance began to rise first and then stabilized. The adhesion on stainless steel surfaces reached up to $27.8 \mathrm{kPa}$ indicating an excellent adhesion performance of the PDA-C-S-PAM hydrogel. In addition, it also exhibited excellent adhesion to hydrophobic (leaf) and hydrophilic (glass) surfaces. The hydrogel could hold two pieces of glass and withstand up to $500 \mathrm{~g}$ in weight. It could also adhere directly to the surface of a person's skin and easily adhere to a weight of $63.18 \mathrm{~g}$ (as shown in Fig. 5(e) and Fig. S6 in the ESM). Therefore, the adhesive strength of the PDA-C-SPAM hydrogel is higher than that of commercially available glue sticks and double-sided tape. 

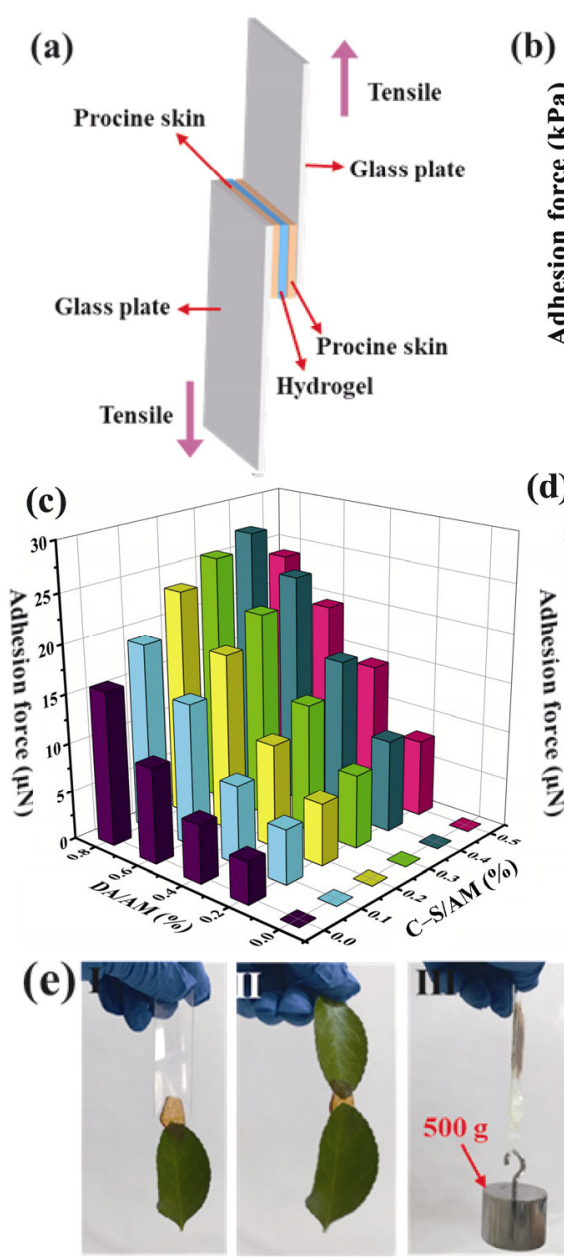

(d)
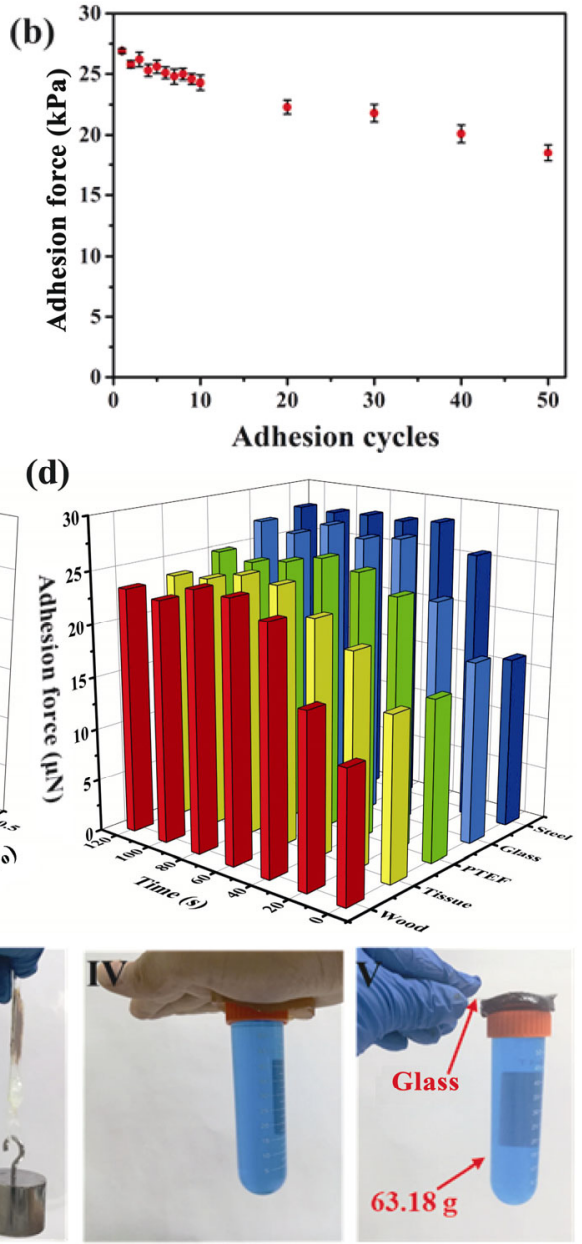

Fig. 5 (a) Adhesion diagram of the hydrogels. (b) Adhesion data of cyclic test materials. (c) Effect of different addition amounts on the adhesion of materials. (d) Effect of surface type and adhesion time on the adhesion of materials. (e) Material can adhere to different infiltrating materials.

The excellent adhesive properties of hydrogels are important in specific biological applications, such as tissue adhesion, cell adhesion, wound dressings, and bone patches. First, the hydrogel adheres very well to the surface of the skin and remains stable with the rotation of the arm (as shown in Fig. 6(a)), allowing for a rotation from $-40^{\circ}$ to $45^{\circ}$ without any signs of debonding. Second, this hydrogel does not show any residue or allergy symptoms when and after it adheres to the skin of the human body (as shown in Fig. 6(b)). It exhibits excellent tissue adhesion (Fig. 6(c)) and requires a large external force to be peeled off. In addition, the continuous adhesion of the hydrogel material is also important for its application, as shown in Fig. 6(d). Here, we cut a piece of hydrogel sample from the middle and tested the coherent adhesion between the two.
We found that it still has excellent adhesion at the hydrogel cut. Notably, we also found that this hydrogel exhibits some extent of self-healing property. After the two parts from the cut are spliced together for $2 \mathrm{~h}$, the hydrogel could heal, retaining nearly $80 \%$ of the original strength (as shown in Fig. S7 in the ESM). This excellent adhesion and self-healing capability of the hydrogels afford potential applications in the field of biology, among others. This excellent adhesion of hydrogels is due to the multiple interactions between the interface and the material, as shown in Fig. S8 in the ESM. The $\mathrm{N}-\mathrm{H}$ and $-\mathrm{NH}_{2}$ groups of PDA, chitosan, and potato residues can form hydrogen bonds with the $\mathrm{N}, \mathrm{O}$, and $\mathrm{F}$ components of solid materials to improve adhesion. In addition, due to the unsaturated heterocyclic structure of polydopamine and potato residue, there 
may be other interactions between the hydrogel and the solid surface, such as $\pi-\pi$ conjugation and hydrophobic interactions. Furthermore, we tested the effect of temperature on the adhesion performance of the hydrogel. Figure S9 in the ESM shows the adhesion performance of the hydrogel stored at different temperatures from -4 to $65{ }^{\circ} \mathrm{C}$. Figure S9(a) in the ESM shows that the hydrogel can still adhere directly to the leaves after being stored at $-4{ }^{\circ} \mathrm{C}$ for $24 \mathrm{~h}$, but the hydrogel loses its adhesion property after being heated at $65{ }^{\circ} \mathrm{C}$ for $24 \mathrm{~h}$ (as shown in Fig. S9(b)). Figure S9(c) in the ESM shows the adhesion performance of hydrogel after storage at various temperatures $\left(-4-65{ }^{\circ} \mathrm{C}\right)$. This result indicates that the material can withstand a certain range of temperatures.

\subsection{In vitro antibacterial Test}

Bacterial infections on the wound site trigger inflammation or immune response hindering wound healing. Antimicrobial activity is one of the most important characteristics of a hydrogel used as a wound dressing. Antibacterial activity of the PDA-C-SPAM hydrogel was evaluated against Staphylococcus epidermidis (Gram-positive bacterium). Images of bacterial suspensions co-cultured with hydrogels

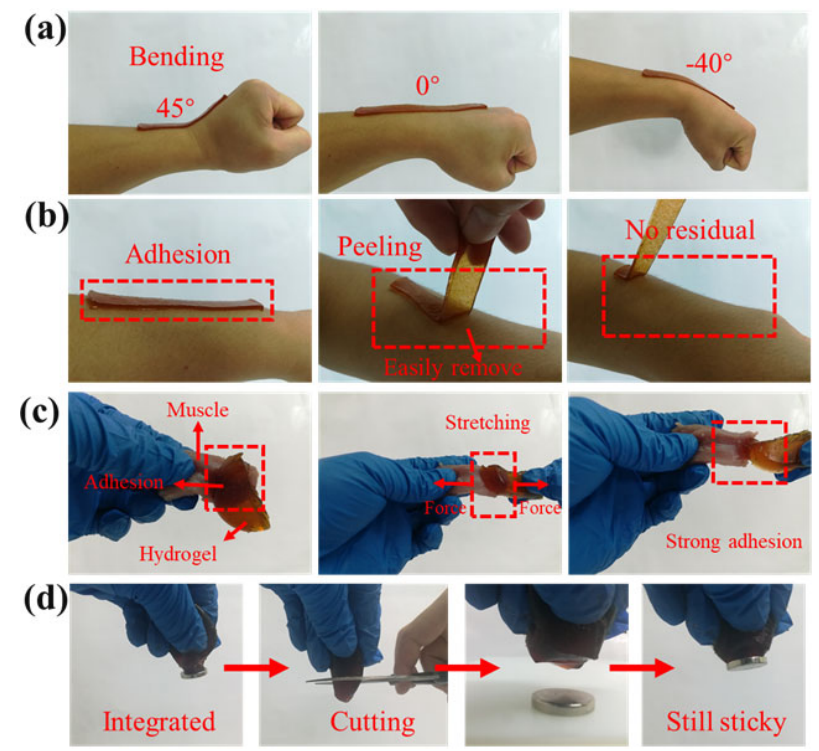

Fig. 6 Material adhering to the skin surface (a) when bending the arm and (b) without leaving any residue after being peeled off. (c) Material can adhere to the muscle tissue. (d) Adhesion of materials remains coherent. are presented in Fig. 7(a). Compared with the blank group, the suspensions of the PDA-C-S-PAM hydrogel were clear because of the presence of dopamine hydrochloride in the hydrogel. As shown in Fig. 7(b), the PDA-C-S-PAM hydrogel had an excellent antibacterial effect. Quantitative analysis showed that the antibacterial rate of the PDA-C-S-PAM hydrogel against $E$. coli was $81.6 \%$ (as shown in Fig. $7(\mathrm{~b})$ ). The results indicate that the PDA-C-S-PAM hydrogel has a good antibacterial effect. This effect may be due to the dopamine destroying the bacterial cell wall and promoting the antibacterial activity of the hydrogel. Fibroblast culture experiments showed that the PDA-C-S-PAM hydrogel had no significant effect on the adhesion and proliferation of fibroblasts after $24 \mathrm{~h}$ of culture. As shown in Fig. 7(c), the fibroblasts on the PDA-C-S-PAM hydrogel grew well, and the cell morphology was normal. The cells also spread well with filamentous pseudopodia, and the number was close to that of the control group. The quantitative analysis results in Fig. 7(d) show that after $24 \mathrm{~h}$ of culture, fibroblasts proliferated on the PDA-C-S-PAM hydrogel. The prepared gel had low toxicity to cells, and the cell survival rate

(a)

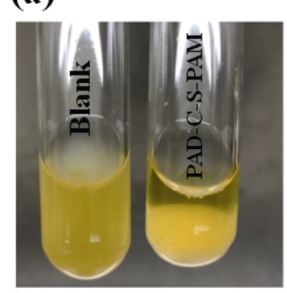

(c)

(b)
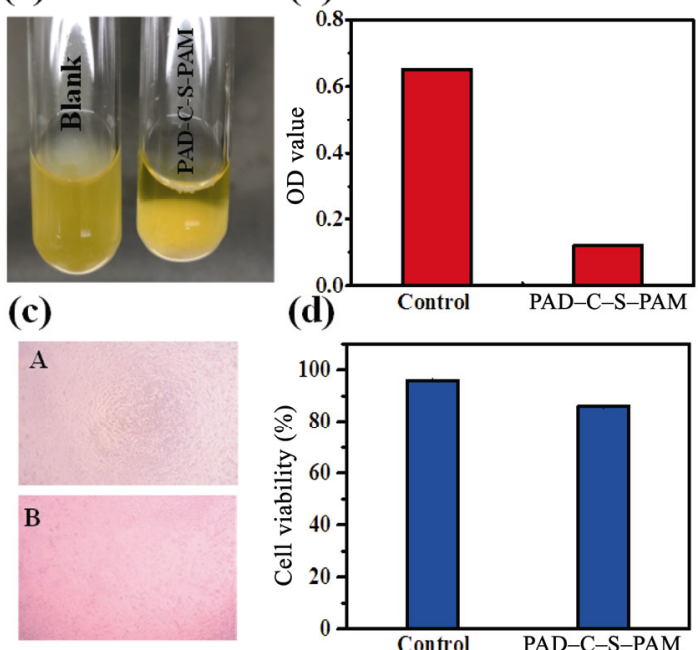

(d)

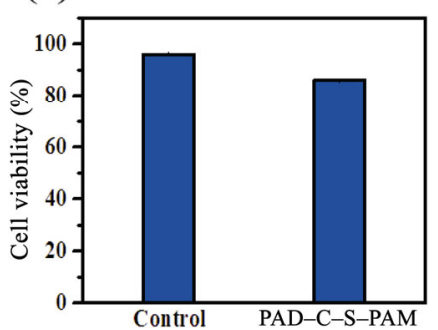

Fig. 7 Antibacterial activity of the PDA-C-S-PAM hydrogel. (a) E. coli suspensions that were cultured with different specimens for 24 h. (b) Optical density (OD) measurement of growth $E$. coli that were cultured with different specimens for $24 \mathrm{~h}$. (c) Comparison of rat fibroblasts and hydrogels co-cultured for $24 \mathrm{~h}$ (A: control; B: PDA-C-S-PAM). (d) Survival rate of rat fibroblasts treated with PDA-C-S-PAM hydrogel. 
was above $85 \%$. The excellent antibacterial effect of hydrogel promotes its potentiality in biological applications.

\subsection{Electrical properties of hydrogels}

The PDA-C-S-PAM hydrogel can be used as an electrical conductor due to its excellent electrical conductivity. Hydrogel has excellent adhesion and can adhere directly to the skin surface to test the pulse signal (Fig. S10 in the ESM). As shown in Fig. 8(a), the PDA-C-S-PAM hydrogel has excellent conductivity and can illuminate a light emitting diode (LED). At the same time, the broken hydrogel can still conduct electricity and illuminate the LED even after self-healing. After stretching the hydrogel multiple times, its resistivity changed regularly with the increase in the number of stretching cycles (as shown in Fig. 8(b)), indicating that the conductivity of the hydrogel can be adjusted by the stretching material. In addition, the ability to deliver a high voltage current has been demonstrated.

As shown in Figs. 8(c) and 8(d), based on the material's good electrical conductivity and adhesion properties, we developed a remote monitoring device with a gauge factor of 1.6 for body signals. As displayed in Fig. 8(e), the hybrid hydrogel was placed over the radial artery to measure the pulse rate, where the frequency of the blood pulse was determined by the frequency of resistance change. Under relaxed conditions, the pulse rate was calculated to be 80 beats/min. Figure $8(f)$ shows that output electrical signal changes with the degree of finger bending and waveform peaks are relatively stable. We also compared the various properties of hydrogels with those of the findings of other studies (as shown in Table S2 in the ESM). We found that the hydrogel demonstrates high performance thereby contributing to its broad application value. Based on the above demonstrations of human motion monitoring, the hybrid hydrogel shows great potential to be used in fabricating wearable devices for monitoring full-range human activities with high sensitivity and reliability.

(b)

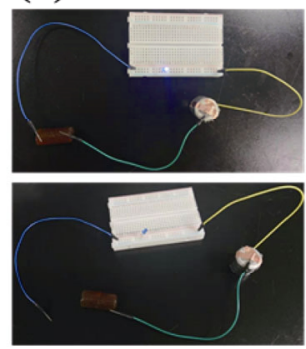

(c)
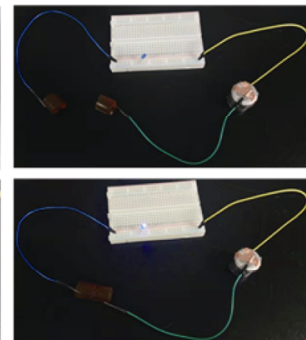

8

Phone

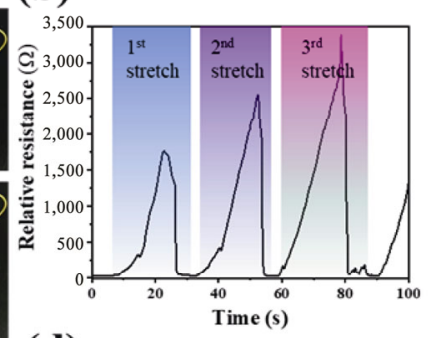

(d)
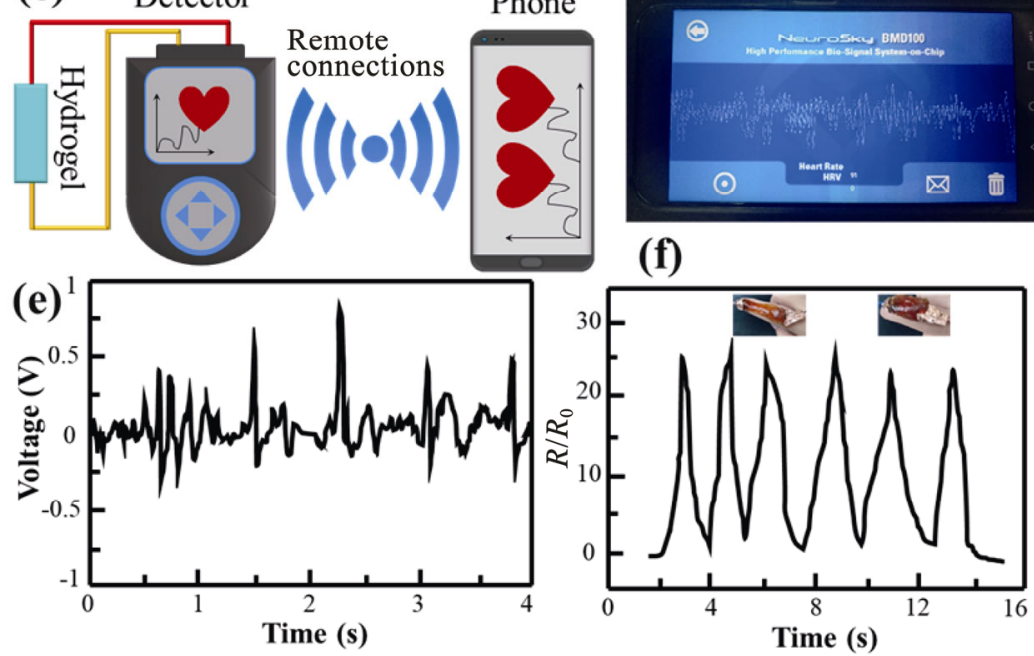

Fig. 8 (a) Conductivity of materials. Material can illuminate LED lights. (b) Resistance of the material exhibits a certain regularity under conditions of tension and compression. (c) Remote monitoring of body signals with the adhesion and (d) conductivity of materials. (e) The heartbeat signal data obtained by the test. (f) The electrical signal data monitored when the finger is bent. 


\section{Conclusions}

In this study, we have developed a facile and environmentally friendly strategy for preparing super-tough, recoverable, self-healing, and highly conductive adhesive hydrogels by in situ freeradical polymerization of waste potato residues, chitosan, and AM. The presence of chitosan and waste potato residues provides the framework of the hydrogel with a perfect environment to maintain excellent mechanical properties. The hydrogel displays a mechanical strength of $0.75 \mathrm{MPa}$ and a fracture strain of more than $900 \%$. Remarkably, the PDA-CS-PAM hydrogel shows self-healing characteristics without any external incitation and an excellent reversible adhesive feature ( $27 \mathrm{kPa})$ with various substrates even on the human skin. Moreover, the hydrogel exhibits ultra-sensitive and stable resistance responsiveness. It can therefore be used as a strain sensor to accurately and reliably monitor various human activities, including large movements of the human body (e.g., finger bending) and the subtle movements of the muscles (such as pulse beats) indicating that they have a great potential in wearable device applications. Hydrogel also has excellent antibacterial effects and is expected to be used as a postoperative healing material, thereby boosting its application in the field of biomedicine. In summary, PDA-C-S-PAM hydrogels have broad application prospects in the fabrication of electronic skin, bionic robots, and wearable smart devices.

\section{Acknowledgements}

This work was supported by the National Natural Science Foundation of China (Nos. 51875577 and 81972901), Tribology Science Fund of State Key Laboratory of Tribology (No. SKLTKF16A06), Chinese PLA General Hospital Training Program "The National Science Found for Distinguished Young Scholars" (No. 2019-JQPY-004), The Science Foundation of China University of Petroleum-Beijing (Nos. 2462019QNXZ02 and 2462018BJC004). T.S.H. acknowledges the support from the U.S. National Science Foundation PREM Program (NSF DMR-1523588).
Electronic Supplementary Material: Supplementary material is available in the online version of this article at https://doi.org/10.1007/s40544-020-0416-x.

Open Access: This article is licensed under a Creative Commons Attribution 4.0 International License, which permits use, sharing, adaptation, distribution and reproduction in any medium or format, as long as you give appropriate credit to the original author(s) and the source, provide a link to the Creative Commons licence, and indicate if changes were made.

The images or other third party material in this article are included in the article's Creative Commons licence, unless indicated otherwise in a credit line to the material. If material is not included in the article's Creative Commons licence and your intended use is not permitted by statutory regulation or exceeds the permitted use, you will need to obtain permission directly from the copyright holder.

To view a copy of this licence, visit http://creativecommons.org/licenses/by/4.0/.

\section{References}

[1] Sun X M, Lang Q, Zhang H B, Cheng L Y, Zhang Y, Pan G Q, Zhao X, Yang H L, Zhang Y G, Santos H A, et al. Electrospun photocrosslinkable hydrogel fibrous scaffolds for rapid in vivo vascularized skin flap regeneration. Adv Funct Mater 27(2): 1604617 (2017)

[2] Sun J Y, Zhao X H, Illeperuma W R K, Chaudhuri O, Oh K H, Mooney D J, Vlassak J J, Suo Z G. Highly stretchable and tough hydrogels. Nature 489(7414): 133-136 (2012)

[3] Trung T Q, Lee N E. Recent progress on stretchable electronic devices with intrinsically stretchable components. Adv Mater 29(3): 1603167 (2017)

[4] Lin S T, Yuk H, Zhang T, Parada G A, Koo H, Yu C J, Zhao X H. Stretchable hydrogel electronics and devices. Adv Mater 28(22): 4497-4505 (2016)

[5] Rim Y S, Bae S H, Chen H J, De Marco N, Yang Y. Recent progress in materials and devices toward printable and flexible sensors. Adv Mater 28(22): 4415-4440 (2016)

[6] Liu W, Song M S, Kong B, Cui Y. Flexible and stretchable energy storage: Recent advances and future perspectives. Adv Mater 29(1): 1603436 (2017)

[7] Feig V R, Tran H, Lee M, Bao Z N. Mechanically tunable conductive interpenetrating network hydrogels that mimic the elastic moduli of biological tissue. Nat Commun 9(1): 2740 (2018) 
[8] Boyer C, Figueiredo L, Pace R, Lesoeur J, Rouillon T, Le Visage C, Tassin J F, Weiss P, Guicheux J, Rethore G J. Laponite nanoparticle-associated silated hydroxypropylmethyl cellulose as an injectable reinforced interpenetrating network hydrogel for cartilage tissue engineering. Acta Biomater 65: 112-122 (2018)

[9] Gong Z Y, Zhang G P, Zeng X L, Li J H, Li G, Huang W P, Sun R, Wong C. High-strength, tough, fatigue resistant, and self-healing hydrogel based on dual physically crosslinked network. ACS Appl Mater Interfaces 8(36): 24030-24037 (2016)

[10] Webber R E, Creton C, Brown H R, Gong J P. Large strain hysteresis and mullins effect of tough doublenetwork hydrogels. Macromolecules 40(8): 2919-2927 (2007)

[11] Zhang H J, Sun T L, Zhang A K, Ikura Y, Nakajima T, Nonoyama T, Kurokawa T, Ito O, Ishitobi H, Gong J P. J. A. M. Tough physical double-network hydrogels based on amphiphilic triblock copolymers. Adv Mater 28(24): 4884-4890 (2016)

[12] Chen F, Tang Z Q, Lu S P, Zhu L, Wang Q L, Gang Q, Yang J, Chen Q. Fabrication and mechanical behaviors of novel supramolecular/polymer hybrid double network hydrogels. Polymer 168: 159-167 (2019)

[13] Zhao Z G, Fang R C, Rong Q F, Liu M J. Bioinspired nanocomposite hydrogels with highly ordered structures. Adv Mater 29(45): 1703045 (2017)

[14] De France K J, Hoare T, Cranston E D. Review of hydrogels and aerogels containing nanocellulose. Chem Mater 29(11): 4609-4631 (2017)

[15] Liu Y, Meng H, Qian Z C, Fan N, Choi W, Zhao F, Lee B P. A moldable nanocomposite hydrogel composed of a mussel-inspired polymer and a nanosilicate as a fit-toshape tissue sealant. Angew Chem Int Ed 56(15): 4224-4228 (2017)

[16] Han L, Lu X, Liu K Z, Wang K F, Fang L M, Weng L T, Zhang $\mathrm{H}$ P, Tang $\mathrm{Y} \mathrm{H}$, Ren $\mathrm{F} \mathrm{Z}$, Zhao $\mathrm{C} \mathrm{C}$, et al. Mussel-inspired adhesive and tough hydrogel based on nanoclay confined dopamine polymerization. ACS Nano 11(3): 2561-2574 (2017)

[17] Han L, Yan L W, Wang K F, Fang L M, Zhang H P, Tang Y H, Ding Y H, Weng L T, Xu J L, Weng J, et al. Tough, self-healable and tissue-adhesive hydrogel with tunable multifunctionality. NPG Asia Mater 9(4): e372 (2017)
[18] Yang P, Zhang S, Chen X F, Liu X H, Wang Z, Li Y W. Recent developments in polydopamine fluorescent nanomaterials. Mater Horiz 7(3): 746-761 (2020)

[19] Sun D T, Peng L, Reeder W S, Moosavi S M, Tiana D, Britt D K, Oveisi E, Queen W L. Rapid, selective heavy metal removal from water by a metal-organic framework/ polydopamine composite. ACS Cent Sci 4(3): 349-356 (2018)

[20] Han L, Liu K Z, Wang M H, Wang K F, Fang L M, Chen $\mathrm{H}$ T, Zhou J, Lu X. Mussel-inspired adhesive and conductive hydrogel with long-lasting moisture and extreme temperature tolerance. Adv Funct Mater 28(3): 1704195 (2018)

[21] Liu X, Zhang Q, Gao Z J, Hou R B, Gao G H. Bioinspired adhesive hydrogel driven by adenine and thymine. ACS Appl Mater Interfaces 9(20): 17645 17652 (2017)

[22] Jing X, Mi H Y, Lin Y J, Enriquez E, Peng X F, Turng L S. Highly stretchable and biocompatible strain sensors based on mussel-inspired super-adhesive self-healing hydrogels for human motion monitoring. ACS Appl Mater Interfaces 10(24): 20897-20909 (2018)

[23] Singh A K, Mishra S, Singh J K. Underwater superoleophobic biomaterial based on waste potato peels for simultaneous separation of oil/water mixtures and dye adsorption. Cellulose 26(9): 5497-5511 (2019)

[24] Fritsch C, Staebler A, Happel A, Márquez M A C, Aguiló-Aguayo I, Abadias M, Gallur M, Cigognini I M, Montanari A, López M J, et al. Processing, valorization and application of bio-waste derived compounds from potato, tomato, olive and cereals: A review. Sustainability 9(8): 1492 (2017)

[25] Liu Y L, Ai K L, Lu L H. Polydopamine and its derivative materials: Synthesis and promising applications in energy, environmental, and biomedical fields. Chem Rev 114(9): 5057-5115 (2014)

[26] Xu Q, Xu M, Lin C Y, Zhao Q, Zhang R, Dong X X, Zhang Y D, Tian S C, Tian Y, Xia Z H. Metal coordination-mediated functional grading and self-healing in mussel byssus cuticle. Adv Sci 6(23): 1902043 (2019)

[27] Yang L, Gu B N, Chen Z, Yue Y, Wang W X, Zhang H T, Liu X H, Ren S J, Yang W Q, Li Y W. Synthetic biopigment supercapacitors. ACS Appl Mater Interfaces 11(33): 30360-30367 (2019) 


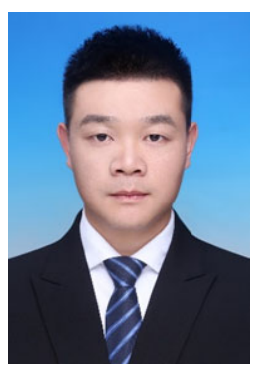

Weijun LI. He received his master's degree from China University of Petroleum (Beijing) in 2020 under Prof. Quan Xu's supervision. He is currently a Ph.D. student in Prof.
Xu Hou' s group at Xiamen University. His research interests focused on bio-inspired smart porous and channel systems, polymer membrane, and hydrogel materials.

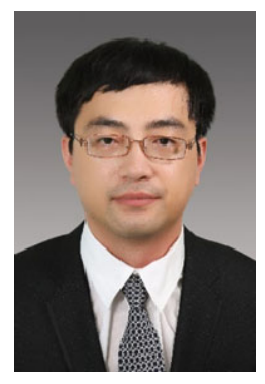

Quan XU. He received his bachelor's

Prof. Zhenhai Xia's supervision. He joined the College degree from the Department of of New Energy and Materials Science, China University Materials Science and Engineering, Shandong University and Ph.D. degree in the Department of Materials Science and Engineering, University of North Texas, under of Petroleum-Beijing in 2014 and now promoted as a full processor. He has over 80 publications with over 2500 citations and his research is related to biomimicry reversible adhesion surfaces and their energy, medical, and environmental applications. 